\title{
Subtotal Colectomy as Part of Debulking Surgery for Advanced-stage Ovarian Cancer
}

\author{
NICOLAE BACALBASA ${ }^{1,2,3}$, CAMELIA DIACONU ${ }^{1,4}$, LAURA ILIESCU $^{1,5}$, SIMONA DIMA $^{3}$, \\ OVIDIU GABRIEL BRATU ${ }^{1,6}$, DRAGOS CRETOIU ${ }^{1,7}$, ADRIAN NEACSU $^{1,8}$, \\ ALEXANDRU FILIPESCU ${ }^{1,9}$, CORNEL SAVU $^{1,10}$ and IRINA BALESCU ${ }^{11}$ \\ 1 "Carol Davila” University of Medicine and Pharmacy, Bucharest, Romania; \\ ${ }^{2}$ Department of Obstetrics and Gynecology, "I. Cantacuzino" Clinical Hospital, Bucharest, Romania; \\ ${ }^{3}$ Department of Visceral Surgery, Center of Excellence in Translational \\ Medicine "Fundeni" Clinical Institute, Bucharest, Romania; \\ ${ }^{4}$ Department of Internal Medicine, University Emergency Hospital Bucharest, Bucharest, Romania; \\ ${ }^{5}$ Department of Internal Medicine, "Fundeni” Clinical Institute, Bucharest, Romania; \\ ${ }^{6}$ Department of Urology, Emergency Central Military Hospital, \\ Academy of Romanian Scientists, Bucharest, Romania; \\ 7 "Alessandrescu-Rusescu” National Institute of Mother and Child Health, \\ Fetal Medicine Excellence Research Center, Bucharest, Romania; \\ ${ }^{8}$ Department of Obstetrics and Gynecology, "St. John” Emergency Clinical Hospital, Bucharest, Romania; \\ ${ }^{9}$ Department of Obstetrics and Gynecology, "Elias" Emergency Hospital, Bucharest, Romania; \\ ${ }^{10}$ Department of Thoracic Surgery, "Marius Nasta” Institute of Pneumonology, Bucharest, Romania; \\ ${ }^{11}$ Department of Surgery, "Ponderas" Academic Hospital, Bucharest, Romania
}

\begin{abstract}
Background/Aim: The presence of extensive lesions of peritoneal carcinomatosis is found in a significant number of cases of extended digestive resection surgery, such as subtotal colectomy. The aim of this study was to report a series of 17 cases that necessitated performing subtotal colectomy as part of cytoreductive surgery for advancedstage ovarian cancer. Patients and Methods: Between 2012 and 2020 subtotal colectomy was associated as part of the debulking effort in 17 cases. Results: The median age at the time of surgery was 59 years, while the initial stage at the time of diagnosis was IIIC in 14 cases, and respectively IV in three cases. Optimal debulking surgery was achieved in all cases. The continuity of the digestive tract was reestablished in 11 cases, while in the remaining 6 cases a terminal ileostomy was performed. Conclusion: Extended digestive tract resections may be needed in certain cases in
\end{abstract}

This article is freely accessible online.

Correspondence to: Irina Balescu, "Ponderas" Academic Hospital, Nicolae Caramfil 85a Street, Bucharest, Romania. Tel: +40 724077709, e-mail: irina.balescu@ ponderas-ah.ro

Key Words: Subtotal colectomy, peritoneal carcinomatosis, ovarian cancer. order to maximize the debulking effort in patients with advanced-stage ovarian cancer.

Digestive tract involvement is a common finding in both benign and malignant conditions such as endometriosis, cervical, endometrial or ovarian cancer (1). However, extended digestive tract involvement is rather associated with the presence of advanced-stage gynecological malignancies such as ovarian or endometrial cancer and usually occurs by the development of peritoneal carcinomatosis due to the peritoneal and subperitoneal spread of the malignant cells (17). Known to be a lax tissular space between the peritoneal folds, the subperitoneal space plays a crucial role for malignant spread from the pelvic area to the abdominal area. This mechanism is responsible for the development of sigmoid involvement in cases presenting left ovarian tumors and respectively right colon invasion in right ovarian tumors (8-9). Furthermore, the apparition of free tumoral cells in the peritoneal fluid will lead to development of disseminated peritoneal seeding; with four predominant sites reported by Meyers et al:: the Douglas pouch, the superior part of the sigmoidian loop, the right paracolic gutter and the right lower quadrant (10). In such conditions, various aspects of colonic involvement such as extrinsic invasion, fixation, circumferential or partial stenosis might be encountered, the colon being, therefore, the most frequently encountered site 
of involvement in advanced-stage ovarian cancer (1, 5-7). Moreover, omental involvement will lead to development of omental cake, which will further lead to transverse colon and even greater curvature invasion through the gastrocolic ligament $(11,12)$. In this respect, in certain patients extended colonic resections are needed in order to maximize the debulking effort and achieve complete cytoreduction.

\section{Patients and Methods}

After obtaining the Ethical Committee approval no 28/2020, data of patients submitted to surgery for advanced-stage ovarian cancer between 2012 and 2020 were retrospectively reviewed. Among these cases we identified 17 patients who were submitted to subtotal colectomy. In all cases debulking surgery to no residual disease (defined as complete cytoreduction) was tempted. All patients were classified according to the International Federation of Obstetrics and Gynecology FIGO staging (13), while postoperative complications were classified according to Dindo-Clavien scale (14).

\section{Results}

The median age at the time of surgery was 59 years (range $=34-78$ years) while the stage at diagnosis according to FIGO stage was IIIC in 14 cases and respectively IV in the remaining three cases. All three cases classified as FIGO stage IV presented parenchimatous hepatic lesions at the time of initial diagnosis. In all cases disseminated lesions of peritoneal carcinomatosis as well as subperitoneal involvement of the right and sigmoidian colon were responsible for the massive involvement of the colonic area. Among the 17 cases, ascites was present in 11 cases, the median volume being of 1,200 $\mathrm{ml}$ (range=500-2,000 ml). Perioperative details are presented in Table I.

As observed from the data presented in Table I, in all cases total hysterectomy with bilateral adnexectomy, total omentectomy and extended lymph node dissection; in addition, extended parietal peritoneal resection was needed in 15 cases due to the presence of synchronous lesions of parietal and pelvic peritoneal nodules (Figures 1-3); moreover, in six cases the presence of invasive nodules of peritoneal carcinomatosis at the diaphragmatic level imposed performing full thickness diaphragmatic resections. In five of the 17 cases mesenteric nodules of carcinomatosis invading the small bowel were encountered and therefore segmental enterectomies were needed. Partial cystectomy was associated in three cases presenting extended lesions of peritoneal carcinomatosis invading the urinary bladder - in one case, and respectively due to the presence of large pelvic masses invading both the sigmoidian loop and the urinary bladder in two cases. The presence of peritoneal nodules at the level of the splenic colonic angle invading the spleen imposed
Table I. Perioperative details of patients submitted to subtotal colectomy as part of primary cytoreduction for advanced-stage ovarian cancer.

\begin{tabular}{lc}
\hline Perioperative characteristics & No of cases \\
\hline FIGO stage & \\
IIIC & 14 \\
IV & 3 \\
Nutritional status: & \\
Albumin level $<3 \mathrm{~g} / \mathrm{dl}$ & 9 \\
Albumin level $>3 \mathrm{~g} / \mathrm{dl}$ & 8 \\
Body mass index & \\
Underweighted $\left(<18 \mathrm{~kg} / \mathrm{m}^{2}\right)$ & 3 \\
Normal $\left(<25 \mathrm{~kg} / \mathrm{m}^{2}\right)$ & 5 \\
Overweighed $\left(25-30 \mathrm{~kg} / \mathrm{m}^{2}\right)$ & 3 \\
Obese $\left(>30 \mathrm{~kg} / \mathrm{m}^{2}\right)$ & 6 \\
Histopathological findings & \\
Serous cell ovarian carcinoma & 15 \\
Clear cell ovarian carcinoma & 2 \\
Degree of differentiation & \\
Well differentiated & 2 \\
Moderately differentiated & 9 \\
Poorly differentiated & 6 \\
Associated resections & \\
Total hysterectomy with bilateral adnexectomy & 17 \\
Total omentectomy & 17 \\
Pelvic and para-aortic lymph node dissection & 17 \\
Peritonectomy & 15 \\
Diaphragmatic resections & 6 \\
Enterectomy & $50 \mathrm{~min}$ \\
Partial cystectomy & 5 \\
Atypical hepatectomy & 3 \\
Splenectomy & 2 \\
Partial gastrectomy & \\
Cholecystectomy & \\
Mean estimated blood loss & \\
& \\
Mean length of surgery & \\
& \\
& \\
& \\
& \\
&
\end{tabular}

association of splenectomy in one case, while the presence of a tumoral mass invading the omentum, the transverse colon and the greater gastric curvature imposed extending the resection at the gastric level by performing a partial gastrectomy. In this respect, debulking surgery to no residual disease was achieved in all cases. When it comes to digestive reconstruction, ileo-rectal anastomosis was used in order to re-establish digestive continuity in 11 cases, while in the remaining six cases a terminal ileostomy was performed. This type of reconstruction was preferred in normal weighted patients presenting normal albumin levels and in whom urinary tract resection was not associated; in all the other cases a terminal ileostomy was the option of choice in order to minimize the risk of postoperative morbidity. In all cases in which anastomosis was the option of choice, the length of the rectal stump was of at least $12 \mathrm{~cm}$; in all the other cases a terminal ileostomy was performed. 


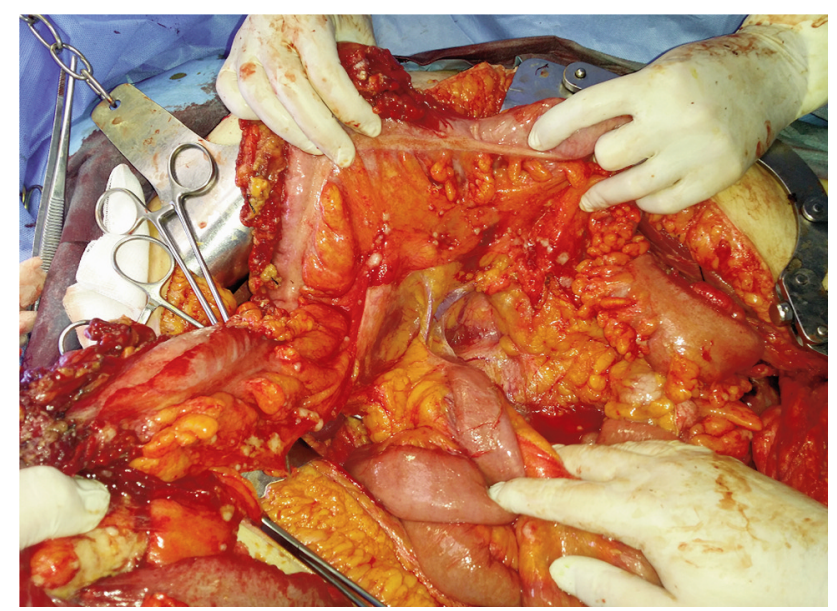

Figure 1. Peritoneal nodules involving the whole length of the colon imposed performing a subtotal colectomy.

During the postoperative period, three cases developed postoperative complications which consisted of the apparition of a pelvic abscess in two cases and respectively one case of postoperative bleeding; one of the two cases diagnosed with a pelvic abscess was submitted to percutaneous drainage and was considered as a DindoClavien grade 3 complication, while the other two cases necessitated reoperation and were therefore considered as grade 4 complications. The median hospital stay was nine days (range $=5-12$ days), postoperatively all patients being confined to the oncology service in order to be submitted to adjuvant oncological treatment.

\section{Discussion}

Complete cytoreductive surgery remains the corner stone in treating advanced-stage ovarian cancer for more than four decades, no other therapeutic strategy can be associated with better long-term outcomes. However, more than half of patients will present colonic involvement at the time of initial diagnosis, imposing performance of digestive tract resections. In this context, although the most commonly encountered site of involvement is represented by the sigmoidian loop, in other cases more extended lesions are present, therefore necessitating more extended resections. Therefore, although rectosigmoidian resection became frequently reported for locally advanced disease, in certain cases other segments of bowel are invaded and required resection $(5-7,15-18)$. In a study conducted on 144 patients with ovarian cancer submitted to surgery with curative intent, $36 \%$ of cases presented extensive colonic involvement excluding the rectosigmoidian site and necessitated more extended colonic resections (19). Among

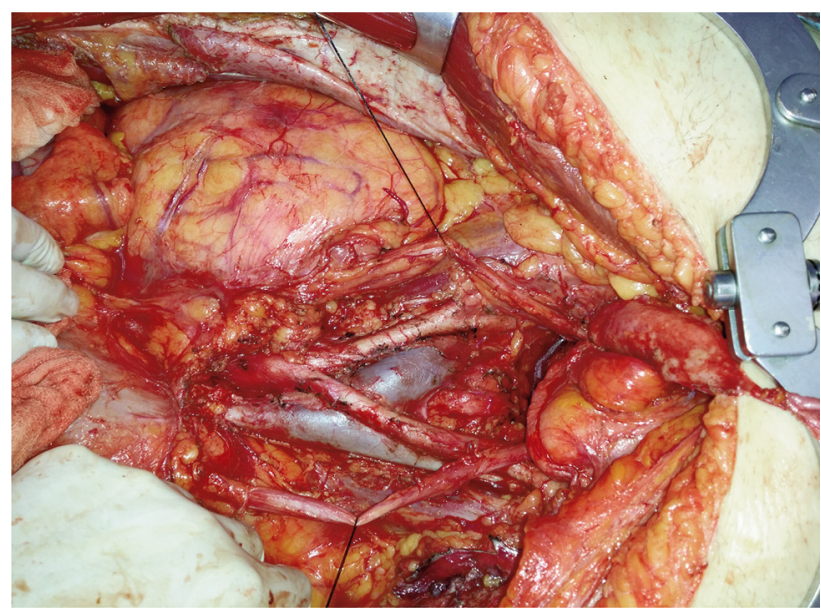

Figure 2. The final aspect after total hysterectomy en-bloc with bilateral adnexectomy, pelvic and para-aortic lymph node dissection, parietal and pelvic peritonectomy and subtotal colectomy.

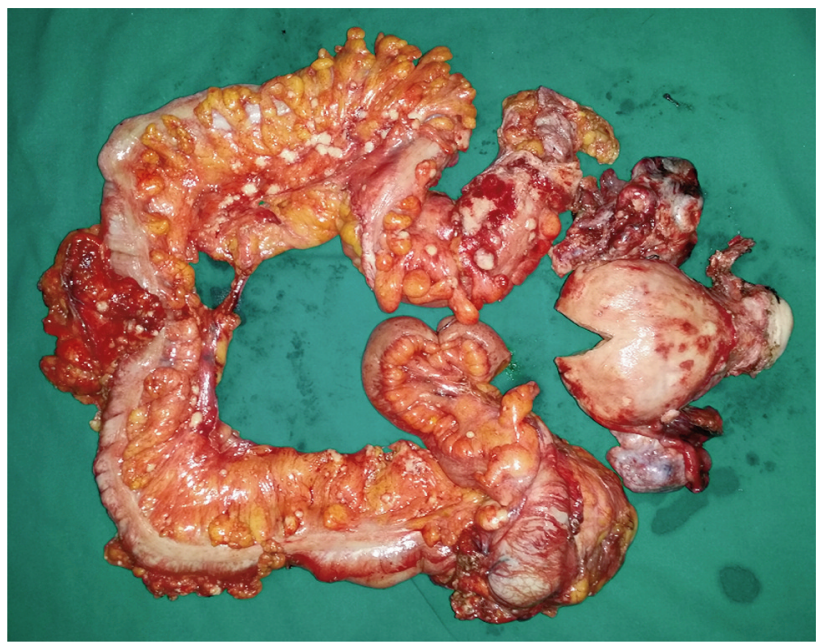

Figure 3. The specimen of total hysterectomy, bilateral adnexectomy and subtotal colectomy.

these there were 17 patients who were submitted to two separate colonic resections, while other five cases presented more extended colonic involvement and necessitated subtotal colectomy. In all cases ileo-rectal anastomosis was associated in order to re-establish the digestive continuity of the digestive tract; this attitude was sustained by the authors' observation regarding the fact that whenever the length of the rectal stump is longer than $12-14 \mathrm{~cm}$, no significant risks of postoperative complications are expected; in this respect the authors have underlined the fact that a single patient developed persistent diarrhea and rectal incontinence (19). 
However, in the study we presented, the decision of performing digestive tract reconstruction via ileo-rectal anastomosis was not taken in cases presenting extended pelvic resections, such as urinary bladder resection or poor nutritional status, due to the high risk of postoperative complications in such cases.

In another more recent study which investigated the necessity of performing extended colonic resections as part of debulking surgery for advanced-stage ovarian cancer, Silver et al. included 19 cases, four of them being submitted to subtotal colectomy; in all cases a $\mathrm{J}$ pouch was used in order to reestablish the continuity of the digestive tract; among these cases, a single patient developed a severe dehydration syndrome, in all the other cases the postoperative course was uneventful (20).

The extension of disease via the gastrocolic ligament and frenocolic ligament will lead to the necessity of extending the field of resection in the left upper abdomen in order to achieve complete debulking. Therefore, in such cases en-bloc left abdominal quadrant resections are performed, the most commonly associated resections being represented by the spleen and the greater gastric curvature. Moreover, in more advanced stages, distal pancreatectomy is needed. In this respect, Hoffman et al. conducted a study on six women submitted to extended left upper abdominal quadrant resections, subtotal colectomy being associated in two cases and were associated with partial pancreatectomy and partial gastrectomy in one case and respectively partial pancreatectomy, partial gastrectomy and left ureteral resection in the second case; in both cases the authors chose to perform an ileo-rectal anastomosis and reported satisfactory outcomes (the only complain being related to the apparition of frequent and semisoft stools - up to eight stools per day) (21).

Subtotal colectomy has been also successfully associated to even more aggressive surgical approaches, such as cytoreduction in association with hyperthermic intraperitoneal chemotherapy, in order to improve the long-term outcomes. Therefore, in a study conducted by Cascales et al. published in the European Journal of surgical Oncology in 2014, among the 91 patients included in the study, subtotal colectomy was needed in three of the 45 cases which necessitated bowel resections (therefore accounting for $3 \%$ of all cases) (22).

An interesting study which investigated the feasibility of extended colonic resections among patients diagnosed with advanced-stage gynecological cancer comes from Korean surgeons conducted by Song et al. (23); the authors conducted a study on 22 patients diagnosed with advancedstage Mullerian cancer submitted to surgery at the National Cancer Center in Korea between 2003 and 2007; among these cases optimal cytoreduction was achieved in 20 cases, the most commonly associated visceral resections being represented by splenectomy in $31.8 \%$ of cases, small bowel resection in $22.7 \%$ of cases and respectively liver resections in $13.6 \%$ of cases. As for the origin of the primary tumor, ovarian cancer was the most commonly encountered malignancy, being reported in $72.7 \%$ of cases. Postoperatively there was no case of anastomotic leak while the fluid loss via the digestive route was successfully controlled by medical treatment; among the 20 cases in a single case a terminal ileostomy was performed while in other two cases a prophylactic ileostomy was associated (23).

As for the opportunity to reestablish the continuity of the digestive tract by performing a ileo-rectal anastomosis, it seems that this method should be preferred in cases in which the rectal stump has a length of $12-14 \mathrm{~cm}$ in order to prevent the apparition of uncontrollable stools and fecal incontinence; whenever a shorter rectal stump is encountered the option of choice should be represented by ileal pouch or terminal ileostomy (24). However, in our study ileal pouch was not performed due to the fact that cases in which a shorter length of the rectal stump was encountered also associated a poorer nutritional status (defined by a lower level of serum albumin) or also associated a segmental resection of the urinary tract, increasing in this way the risk of perioperative morbidity.

\section{Conclusion}

Although the most commonly encountered involved digestive segment is represented by the sigmoidian loop, patients diagnosed in advanced stages of the disease might need more extended resections. In this respect, subtotal colectomy might be needed in order to achieve complete debulking surgery and minimize the postoperative risks, which could be induced by performing multiple colonic anastomoses. Moreover, in certain cases other upper abdominal resections might be included as part of the debulking effort.

\section{Conflicts of Interest}

The Authors have no conflicts of interest to declare regarding this study.

\section{Authors' Contributions}

NB performed the surgical procedures; IB prepared the manuscript; CD, LI, DC, SD perfrormed data analysis; OGB, AF, AN, CS were part of the surgical team; DC advised about the oncological outcome; NB revised the final draft of the manuscript.

\section{Acknowledgements}

This work was supported by the project entitled "Multidisciplinary Consortium for Supporting the Research Skills in Diagnosing, Treating and Identifying Predictive Factors of Malignant Gynecologic Disorders", project number PN-III-P1-1.2PCCDI2017-0833. 


\section{References}

1 Szucs RA and Turner MA: Gastrointestinal tract involvement by gynecologic diseases. Radiographics 16(6): 1251-1270, 1996. PMID: 8946534. DOI: 10.1148/radiographics.16.6.8946534

2 Bacalbasa N, Balescu I, Dima S and Popescu I: Ovarian sarcoma carries a poorer prognosis than ovarian epithelial cancer throughout all FIGO stages: a single-center case-control matched study. Anticancer Res 34(12): 7303-7308, 2014. PMID: 25503164.

3 Bacalbasa N, Balescu I and Dima S: Rectosigmoidian involvement in advanced-stage ovarian cancer - intraoperative decisions. In Vivo 31(5): 973-977, 2017. PMID: 28882968. DOI: 10.21873/invivo. 11156

4 Bacalbasa N, Balescu I and Filipescu A: Debulking surgery for high-grade serous endometrial cancer with disseminated peritoneal lesions. In Vivo 31(4): 719-722, 2017. PMID: 28652446. DOI: 10.21873 /invivo.11120

5 Bacalbasa N, Balescu I, Dima S, Herlea V, David L, Brasoveanu $\mathrm{V}$ and Popescu I: Initial incomplete surgery modifies prognosis in advanced ovarian cancer regardless of subsequent management. Anticancer Res 35(4): 2315-2320, 2015. PMID: 25862895.

6 Bacalbasa N, Balescu I, Dragan I, Banceanu G, Suciu I and Suciu N: Endometrial adenocarcinoma presenting as hematometra with underlying thickened endometrial lining in a postmenopausal woman - A case report. Anticancer Res 36(5): 2353-2357, 2016. PMID: 27127143.

7 Bacalbasa N, Dima S, Balescu I, David L, Brasoveanu V and Popescu I: Results of primary cytoreductive surgery in advancedstage epithelial ovarian cancer: A single-center experience. Anticancer Res 35(7): 4099-4104, 2015. PMID: 26124361.

8 Oliphant M, Berne AS and Meyers MA: Imaging the direct bidirectional spread of disease between the abdomen and the female pelvis via the subperitoneal space. Gastrointest Radiol 13(4): 285-298, 1988. PMID: 3049207. DOI: 10.1007/bf01889084

9 Oliphant M, Berne AS and Meyers MA: Bidirectional spread of disease via the subperitoneal space: the lower abdomen and left pelvis. Abdom Imaging 18(2): 117-125, 1993. PMID: 8439749. DOI: $10.1007 /$ bf00198047

10 Meyers MA: Intraperitoneal spread of malignancies and its effect on the bowel. Clin Radiol 32(2): 129-146, 1981. PMID: 7214829. DOI: 10.1016/s0009-9260(81)80149-3

11 Pombo F, Rodriguez E, Martin R and Lago M: CT-guided coreneedle biopsy in omental pathology. Acta Radiol 38(6): 978-981, 1997. PMID: 9394652. DOI: 10.1080/02841859709172113

12 Souza FF, Mortele KJ, Cibas ES, Erturk SM and Silverman SG: Predictive value of percutaneous imaging-guided biopsy of peritoneal and omental masses: results in 111 patients. AJR Am J Roentgenol 192(1): 131-136, 2009. PMID: 19098191. DOI: 10.2214/AJR.08.1283

13 FIGO Ovarian Cancer Staging, Effective Jan. 1, 2014. Available at: https://www.sgo.org/wp-content/uploads/2012/09/FIGO-OvarianCancer-Staging_1.10.14.pdf [Last accessed on May 11, 2020]

14 Clavien PA, Barkun J, de Oliveira ML, Vauthey JN, Dindo D, Schulick RD, de Santibanes E, Pekolj J, Slankamenac K, Bassi C, Graf R, Vonlanthen R, Padbury R, Cameron JL and Makuuchi $\mathrm{M}$ : The Clavien-Dindo classification of surgical complications: five-year experience. Ann Surg 250(2): 187-196, 2009. PMID: 19638912. DOI: 10.1097/SLA.0b013e3181b13ca2
15 Kuhn W, Florack G, Roder J, Schmalfeldt B, Pache L, Rust M, Ulm K, Spathe k, Janicke F, Rudiger Siewert J and Graeff H: The influence of upper abdominal surgery on perioperative morbidity and mortality in patients with advanced ovarian cancer FIGO III and FIGO IV. Int J Gynecol Cancer 8(1): 56-63, 1998. DOI: $10.1046 / j .1525-1438.1998 .09776 . x$

16 Eisenkop SM and Spirtos NM: Procedures required to accomplish complete cytoreduction of ovarian cancer: is there a correlation with "biological aggressiveness" and survival? Gynecol Oncol 82(3): 435-441, 2001. PMID: 11520137. DOI: $10.1006 /$ gyno.2001.6313

17 Eisenkop SM, Nalick RH and Teng NN: Modified posterior exenteration for ovarian cancer. Obstet Gynecol 78(5 pt 1): 879885, 1991. PMID: 1923216.

18 Bridges JE, Leung Y, Hammond IG and McCartney AJ: En bloc resection of epithelial ovarian tumors with concomitant rectosigmoid colectomy: the KEMH experience. Int J Gynecol Cancer 3(4): 199-202, 1993. PMID: 11578345. DOI: 10.1046/ j.1525-1438.1993.03040199.x

19 Hoffman MS, Griffin D, Tebes S, Cardosi RJ, Martino MA, Fiorica JV, Lockhart JL and Grendys EC Jr.: Sites of bowel resected to achieve optimal ovarian cancer cytoreduction: implications regarding surgical management. Am J Obstet Gynecol 193(2): 582-586, 2005. PMID: 16098902. DOI: 10.1016/j.ajog.2005.03.046

20 Silver DF and Bou ZN: Extended left colon resections as part of complete cytoreduction for ovarian cancer: tips and considerations. Gynecol Oncol 114(3): 427-430, 2009. PMID: 19555997. DOI: 10.1016/j.ygyno.2009.05.037

21 Hoffman MS, Tebes SJ, Sayer RA and Lockhart J: Extended cytoreduction of intraabdominal metastatic ovarian cancer in the left upper quadrant utilizing en bloc resection. Am J Obstet Gynecol 197(2): 209-204, 2007. PMID: 17689654. DOI: 10.1016/j.ajog.2007.04.049

22 Cascales CP, Gil J and Parrilla P: Morbidity and mortality outcomes of cytoreductive surgery and hyperthermic intraperitoneal chemotherapy in patients with primary and recurrent advanced ovarian cancer. Eur J Surg Oncol 40(8): 970-975, 2014. PMID: 24035502. DOI: 10.1016/j.ejso.2013.08.013

23 Song YJ, Lim MC, Kang S, Seo SS, Park JW, Choi HS and Park SY: Total colectomy as part of primary cytoreductive surgery in advanced Mullerian cancer. Gynecol Oncol 114(2): 183-187, 2009. PMID: 19427682. DOI: 10.1016/j.ygyno.2009.04.009

24 Hoffman MS and Zervose E: Colon resection for ovarian cancer: intraoperative decisions. Gynecol Oncol 111(2 suppl): S56-S65, 2008. PMID: 24035502. DOI: 10.1016/j.ejso.2013.08.013
Received April 8, 2020

Revised April 21, 2020

Accepted May 11, 2020 